PALEO

Revue d'archéologie préhistorique

21 | 2009-2010

Varia

\title{
Une nouvelle représentation feminine à la Madeleine (Tursac, Dordogne)
}

Jean-Pierre Duhard

\section{OpenEdition}

1 Journals

Édition électronique

URL : http://journals.openedition.org/paleo/1776

DOI : $10.4000 /$ paleo. 1776

ISSN : 2101-0420

Éditeur

SAMRA

\section{Édition imprimée}

Date de publication : 1 mars 2011

Pagination : 127-134

ISSN : 1145-3370

\section{Référence électronique}

Jean-Pierre Duhard, « Une nouvelle représentation feminine à la Madeleine (Tursac, Dordogne) »,

PALEO [En ligne], 21 | 2009-2010, mis en ligne le 21 octobre 2011, consulté le 07 juillet 2020. URL :

http://journals.openedition.org/paleo/1776 ; DOI : https://doi.org/10.4000/paleo.1776

\section{(c) (i) () $\Theta$}

PALEO est mis à disposition selon les termes de la licence Creative Commons Attribution - Pas d'Utilisation Commerciale - Pas de Modification 4.0 International. 


\title{
UNE NOUVELLE REPRÉSENTATION FÉMININE à La Madeleine (Tursac, Dordogne)
}

\author{
Jean-Pierre DUHARD ${ }^{(1)}$
}

\begin{abstract}
Résumé : L'auteur présente une figure féminine sculptée sur bois de renne, provenant du Magdalénien IV du grand abri de La Madeleine en Dordogne. Elle vient compléter le petit corpus de figurations humaines de ce site, par ailleurs riche en œuvres d'art. Le corps est représenté en vue frontale et se résume à un tronc et à un pelvis, avec indication des racines crurales et du triangle pubo-génital. Dans sa morphologie, il n'est pas sans rappeler la figure gravée de Lespugue et la figure en ronde bosse de Laugerie-Basse et il illustre la tendance au schématisme de ces figures féminines mobilières magdaléniennes.
\end{abstract}

Mots-clés : Paléolithique, Magdalénien, art mobilier, sculpture, figure féminine, La Madeleine.

Key-words: Palaeolithic, Magdalenian, mobiliar art, sculpture, female figure, Madeleine.

\section{Abridged english version}

\section{New female representation in La Madeleine (Tursac, Dordogne).}

The author presents a segment of cortical reindeer antler, elongated and of semi-circular section, carrying a female carved figure from the Magdalenian IV of the large shelter of Madeleine in Dordogne (France) and stored in the Musée national de préhistoire. Discovered by D. Peyrony during his excavations between 1910 and 1913, it had been described in the monography of E. Capitan and D. Peyrony (1928) as a blade dagger (fig. 1). Without prejudice to the use of this object, it provides an unambiguous but schematic representation of a woman (fig. 2, 3, 4, 5), Her body is represented in frontal view, and comes down to the trunk and pelvis, with indication of the starting point of the thighs and the pubogenital triangle. There is no breast, or upper limbs or head ; the ornaments of the sides remain undeciphered (fig. 7, 8). Technically, the reindeer antler has been shaped with reduced thickness and a shaping of a half round, reduced in width to clear the trunk and pelvis. Deep furrows were drawn to show the genito-crural and the intercrural space, and lighter lines for the hypogastric path and the beginning of the vulval cleft (fig. 6). The sides were decorated with simple lines, bow shaped on the right, and linear on the left (fig. 7, 8). This work complements the small corpus of human figures known to this particular site, also rich in works of art and, more generally, that of the Magdalenian female figures. By its morphology, this female body looks like the figure of Lespugue (fig. 9) and the statuette of the nearby station of Laugerie Basse (fig. 10) and illustrates the evolution from a description style to an elliptical style in the Magdalenian art. As for the meaning given to these gendered (women and men) and genital (vulva and penis) figurative works, as delivered by this deposit, it remains speculative. Their development may reveal a legitimate reproduction concern, as much as a healthy interest in human genital organs. 


\section{L'art mobilier de La Madeleine}

Le grand abri-sous-roche de la Madeleine, commune de Tursac (Dordogne), sur la rive droite de la Vézère, en amont des Eyzies, est le site éponyme du Magdalénien, terme proposé par G. de Mortillet en 1869. C'est un des premiers gisements fouillés en Dordogne dès 1863 par E. Lartet et H. Christy. Après d'autres chercheurs (Girod, Massénat, Rivière), D. Peyrony y reprendra des fouilles méthodiques de 1910 à 1913, qui permirent à l'abbé Breuil de proposer une subdivision du Magdalénien en plusieurs phases. Le site a livré des outils et des objets d'art mobilier en abondance, dispersés dans divers musées, ce qui ne facilite pas leur étude. La reprise des fouilles par J. M. Bouvier entre 1968 et 1983 et les recherches qu'il fit ont permis de reconstituer la provenance topographique des objets les plus remarquables. L'abri a été fréquenté sur une longue période par des groupes successifs qui ont laissé leurs industries, allant du Magdalénien moyen au Magdalénien supérieur, avec de nombreux objets en os, ivoire et bois de renne, harpons et sagaies notamment, souvent très richement décorés, de façon figurative ou non, comme le montrent les planches du Reliquiae Aquitanicae de Lartet et Christy (1865-75). "Toutes les techniques d'expression, tous les thèmes, tous les types de support sont représentés par une centaine d'œuvres à décor figuratif et un nombre bien plus important d'outils décorés et d'œuvres abstraites ", confirme Bouvier (1990).

Dans l'art figuratif, les représentations animales dominent, ce qui est la règle dans l'art paléolithique, tant pariétal que mobilier, avec un riche bestiaire où l'on trouve : cheval (nombreuses têtes gravées), mammouth gravé sur une plaque d'ivoire de mammouth'2, bison, lynx, bouquetin, antilope, isard, renne, cerf (biche), sanglier, poisson, etc. Les figures humaines, moins nombreuses que les animales, occupent cependant une place conséquente dans l'art figuratif humain mobilier paléolithique. Les unes sont des représentations de corps humains, les autres, de parties de corps.

De la « couche inférieure ", qui est du Magdalénien IV (Bouvier 1990), ont été extraits notamment (Capitan et Peyrony 1928) ${ }^{3}$ : un galet pyramidal gravé de deux corps humains ${ }^{4}$, où l'un, porteur d'un sein, est féminin et l'autre de sexe indéterminé, mais probablement masculin (Delporte
1993) ; une gravure sur os d'un « personnage masqué ithyphallique $»^{5}$; deux figures « anthropomorphes » dont I'humanité et le sexe restent à préciser, mais où l'une évoque un embryon humain ou animal ; un « lissoir dont une extrémité est découpée en profil de tête humaine " (Capitan, ibid). En provient également, un phallus non équivoque ${ }^{6}$ en bois de renne "dont le dessin dispense de toute description » (ibid), avec son gland demi découvert par le prépuce. Pour mémoire, cette couche a livré, entre autres œuvres magistrales, " une superbe statuette en bois de renne représentant un bison, la tête retournée, se léchant le flanc " (ibid). Les réserves des musées constituent le plus grand gisement de France, plaisantait A. Leroi-Gourhan (in verbim, G. Delluc), et offrent bien des opportunités de découvertes, à l'image de celle présentée ici. Dans la planche des « objets en os, ivoire et bois de renne diversement décorés " provenant de cette couche (Capitan et Peyrony 1928), un objet en bois de renne de la figure $31,1 a$ et $1 b$, porte une ornementation particulière, qui n'a pas retenu l'attention des auteurs, l'ayant identifié comme une "lame de poignard " (fig. 1) et la décrivant ainsi : elle "était ornée de profonds sillons obliques, symétriques deux à deux, partant du bord et convergeant vers le milieu sans se rencontrer (...). Le manche porte une rainure longitudinale et trois arcs de cercle profondément incisés probablement pour éviter le glissement dans la main (...). Les incisions profondes (...) étaient destinées sans doute à favoriser l'écoulement du sang des animaux blessés».

Notre lecture est sensiblement différente comme le laisse supposer le titre de ce texte; nous y reviendrons plus loin.

Dans la « couche moyenne », qui est du Magdalénien V, les " documents d'art figuratifs sont beaucoup moins nombreux que dans le Magdalénien IV sous-jacent " (Bouvier 1990). En proviennent un petit bâton percé en bois de renne, orné d'une vulve gravée, et peut-être d'un gland pénien, et " une belle tête d'ours brun, léchant le gland d'un phallus qui paraît être en érection ${ }^{8}$. Dans les figures de nature humaine possible, retenons aussi un phallus au gland découvert, "à moins que ce ne fût un bouchon d'outre » sur la figure $39, n^{\circ} 8$ (Capitan ibid), et un os gravé de "trois personnages très stylisés se suivant à la file indienne " sur la figure $42, \mathrm{n}^{\circ} 10$ (ibid). Le sexe de l'humain gravé sur un bâton perforé en bois de renne du MAN,

(2) Cette plaque, trouvée par E. Lartet, apportait la preuve de l'ancienneté de l'humain préhistorique, à l'évidence contemporain d'une espèce animale disparue depuis longtemps.

(3) Les citations non référencées sont tirées de l'ouvrage de L. Capitan et $D$. Peyrony ; sinon l'auteur est cité entre parenthèses.

(4) MAN 76950, $98 \mathrm{~mm}$ de haut.

(5) MNP, $72 \mathrm{~mm}$ de long.

(6) MAN 60347, dépôt au MNP, fiche 682-12 (cliché N. Aujoulat), 101 mm de long.

(7) MAN 56880, dépôt au MNP, fiche 672-12 (cliché N. Aujoulat), 100 mm de long.

(8) MAN 56879, $132 \mathrm{~mm}$ de long (décor similaire à celui d'une baguette demi-ronde trouvée dans l'abri de Longueroche, commune de Plazac, à $4 \mathrm{~km}$ de la Madeleine). 


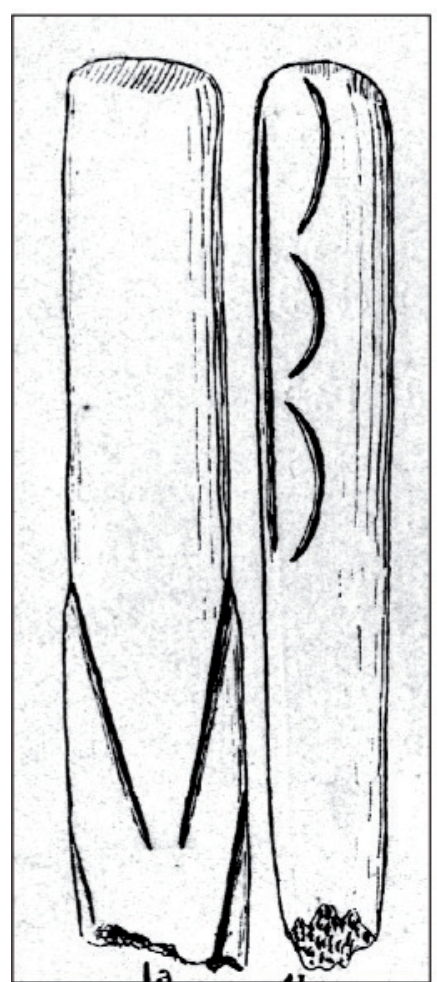

Figure 1 - Relevé de D. Peyrony (Capitan, Peyrony 1928).

Figure 1 - Tracing from D. Peyrony (Capitan, Peyrony 1928).

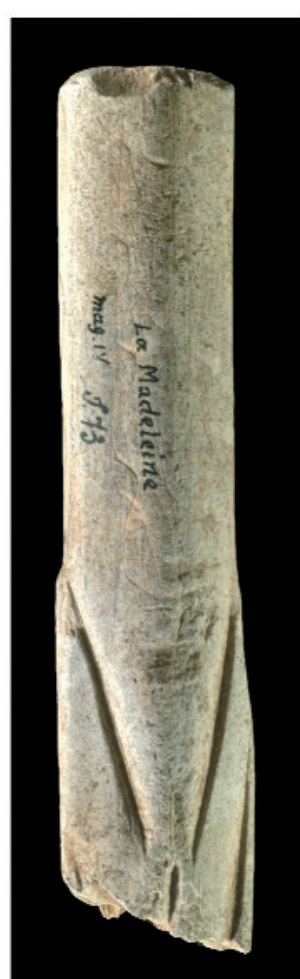

Figure 2 - Face antérieure (os cortical), $131 \mathrm{~mm}$ de long.

Figure 2 - Anterior side (cortical bone), $131 \mathrm{~mm}$ long.

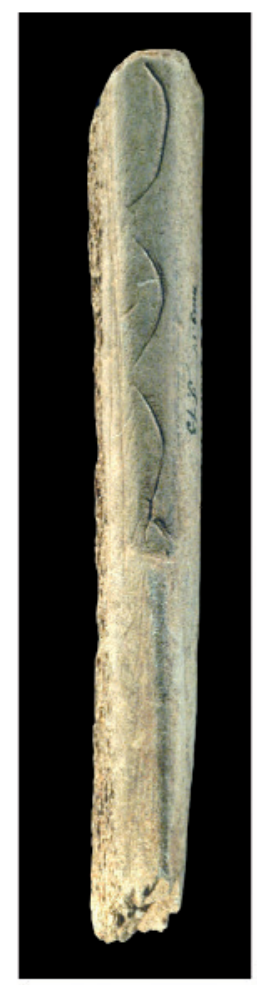

Figure 3 - Face latérale droite avec la frise arciforme.

Figure 3 - Right lateral side with bow form frieze.
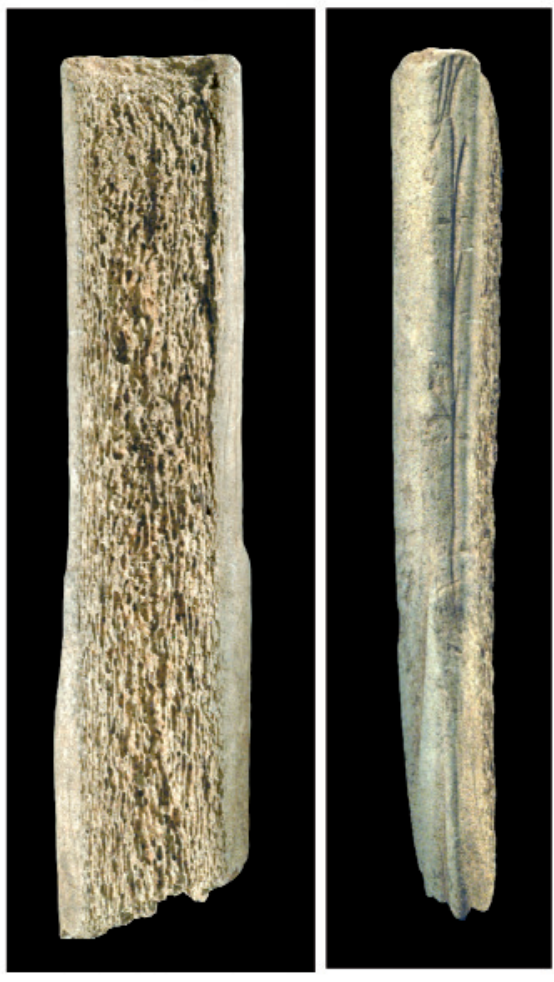

Figure 4 - Face postérieure (os spongieux).

Figure 4 - Back side (spongy bone).
Figure 5 - Face latérale gauche avec le sillon longitudinal.

Figure 5 - Left lateral side with longitudinal groove.

Crédit photo, Ph. Jugie, Musée national de Préhistoire.

Par convention, la face antérieure correspond à la face corticale convexe, la face postérieure à la face spongieuse; le bord droit est celui du corps humain figuré et non pas celui situé à droite de l'image ; de même, pour le gauche.

provenant des fouilles Lartet et Christy ${ }^{9}$ me semble incertain, mais le sujet gravé sur un autre bâton en bois de renne conservé au British museum me paraît bien féminin ${ }^{10}$. Quant aux supposés humains de la planche XVI (ibid), gravés sur une dalle calcaire trouvée dans la cella de l'abri, et rapprochés des personnages opposés de Laussel (coït ou enfantement ?), ils restent énigmatiques et le resteront, tant que la pièce ne sera pas retrouvée. Capitan et Peyrony, comparant les industries de cette couche et de celle sous-jacente, écrivaient : " l'art stylisé, qui avait débuté pendant la phase précédente, se développa de plus en plus et tint une plus grande place que l'art réaliste dans la décoration mobilière » (ibid p. 80-81).
II faut préciser que la " couche supérieure ", du Magdalénien VI, n'a pas livré de figures humaines. La Madeleine n'est pas le site ayant livré le plus grand nombre d'œuvres mobilières magdaléniennes à représentations humaines, mais il vient en bonne place après La Marche et le Roc-aux-Sorciers, et à côté de Laugerie-Basse.

\section{La figure féminine sur segment cortical de bois de renne}

Cet objet, de forme allongée et à section semi-ronde, est exposé dans la vitrine de l'abri de la Madeleine au Musée national de Préhistoire des Eyzies (MNP), et étiqueté

(9) Magdalénien moyen-récent, MAN 8163.

(10) Bâton de 218 mm de longueur (Duhard 1999, pl. XXXV). 
« fragment de ciseau en bois de cervidé » (fig. 2 à 5). Sa nature véritable n'a pas échappée à $\mathrm{N}$. Aujoulat qui, dans son inventaire de l'art mobilier du MNP, l'a dénommé «sculpture anthropomorphe probable ». À juste titre, car il s'agit effectivement d'un humain, et plus précisément d'une figure féminine sculptée et gravée, représentée de face, de la partie supérieure du tronc à la racine des cuisses, et que je vais décrire. Provenant bien du Magdalénien IV, il s'agit d'un segment cortical de bois de renne, allongé et à section semi ronde long de $131 \mathrm{~mm}$, large de $30 \mathrm{~mm}$ et épais de $13 \mathrm{~mm}$, taillé dans un bois de renne, probablement dans le merrain ou tige centrale de la ramure. Le renne devait être un gibier préférentiel car «sur certains points, les bois de renne se trouvaient entassés dans tous les sens et formaient un puissant dépôt » dans la couche inférieure ; et "ils avaient presque tous subi un commencement de débitage ", rapportent les auteurs de la monographie (Capitan et Peyrony 1928).

Le support a été réduit de moitié dans son épaisseur, faisant apparaître l'os spongieux, aux alvéoles étroites et serrées, sur le verso plat de la pièce. L'extrémité terminale de l'objet est régulière, comme si elle avait été sciée horizontalement, et ne porte pas de traces de fracture mais d'écrasement. L'extrémité basale, en apparence brisée (selon une ligne oblique irrégulière de gauche à droite et de haut en bas) semble avoir été régularisée sur l'arête corticale antérieure. Telle qu'elle est, elle n'offre pas de difficultés de lecture. La face corticale d'os compact, qui porte la figure féminine, a été soigneusement polie, avant voire après, gravure des incisions décrites ; elle offre une section convexe et un modelé qui se retrouve dans les différentes parties du corps figurées, tronc, pelvis et cuisses, et permet de donner un volume à la figure féminine, comme l'offrirait une ronde bosse. Les $2 / 3$ supérieurs ont été légèrement réduits dans la largeur, de façon à dégager la partie tronculaire, qui s'avère plus étroite, de la partie inférieure, pelvicrurale, plus large ; de même une réduction de l'épaisseur de l'os compact cortical aboutit à donner moins de saillant au tronc. Le relief résultant de cette différence de largeur et d'épaisseur permet d'indiquer le rebord iliaque et de marquer la séparation entre le tronc et le massif pelvien et crural.

Les deux profonds sillons obliques de dehors en dedans, décrits par Capitan et Peyrony, et partant du bord à l'union des $2 / 3$ supérieurs et du $1 / 3$ inférieur, à hauteur de l'élargissement du support, convergent bien vers le milieu et sans se rencontrer. Ils correspondent anatomiquement aux sillons génito-cruraux et constituent les deux côtés du triangle pubo-génital ; ce triangle est dépourvu de base, mais un sillon peu marqué en " $S$ » couché figure le pli hypogastrique. Le fond des sillons pelvi-cruraux est marqué de stries parallèles, indiquant qu'un outil à pointe étroite a été passé à plusieurs reprises. A droite, le sillon est plus large dans sa partie inférieure, alors que l'inverse s'observe à gauche, laissant supposer que l'outil n'a pas été utilisé dans le même sens des deux côtés : de haut en bas à droite et de bas en haut à gauche, me paraît-il. Cela pourrait montrer la rotation de l'objet à $180^{\circ}$ entre les deux creusements des sillons, m'a fait observer Madame Feruglio. Un sillon, également oblique de dehors en dedans, délimite la racine crurale gauche ; il a le même aspect que le sillon génitocrural droit, plus large en haut qu'en bas, et avec plusieurs stries dans le fond de sa gorge. Les bords de ces sillons ont été abattus, émoussés, pour respecter le galbe du corps. Entre la racine des cuisses, dans la partie médiane, et juste en dessous du

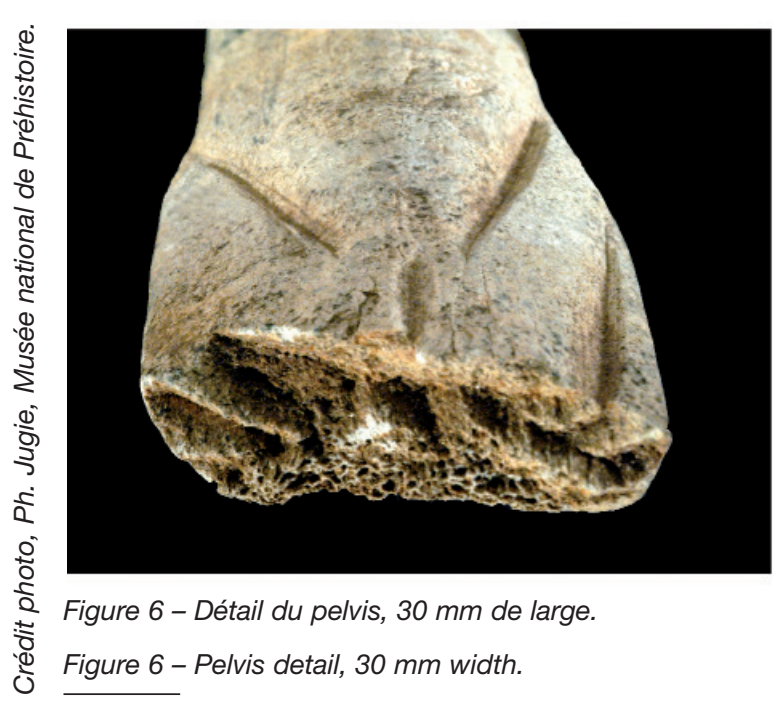

Figure 6 - Pelvis detail, $30 \mathrm{~mm}$ width.

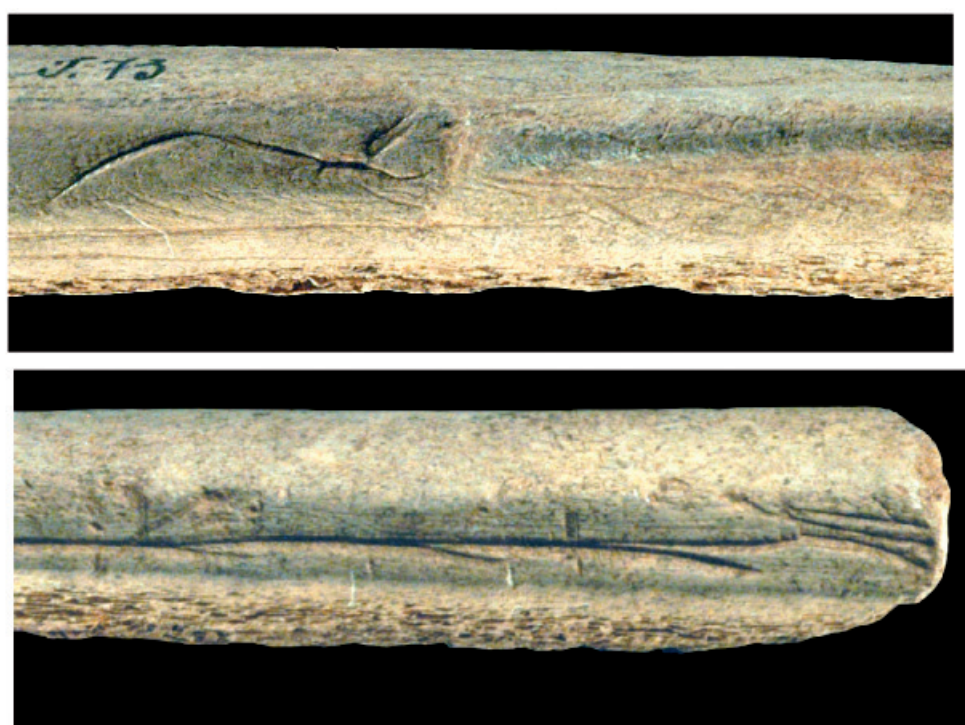

Figure 7 - Détail de la frise arciforme droite vers le pelvis.

Figure 7 - Right bow form detail towards pelvis.

Figure 8 - Détail du sillon longitudinal gauche dans sa partie supérieure.

Figure 8 - Left longitudinal groove detail in its higher part. 
niveau où se terminent les sillons génito-cruraux, un quatrième sillon a été tracé : c'est une rainure courte, étroite, fusiforme et profonde, correspondant anatomiquement à la partie supérieure de l'espace intercrural, quand les cuisses sont en adduction (serrées), ce qui est le cas ici (fig. 6).

La forme ovoïde de ce sillon prêterait à confusion avec une fente vulvaire, s'il n'était situé aussi bas et s'il n'y avait pas une fossette naviforme sus-jacente. En effet, dans l'espace délimité par la partie inférieure convergente, mais non jointive, des sillons génito-cruraux, on observe une dépression longitudinale courte et peu profonde, à fond lisse et plat, bien visible en vue rasante ou tangentielle, qui serait chez le vivant la partie antérieure de l'espace interlabial, la seule partie de la fente vulvaire se voyant sur un sujet debout, en l'absence de pilosité. Une autre hypothèse est à considérer : la fossette serait la partie pubienne de la fente vulvaire résultant de la convergence des grandes lèvres, et le sillon fusiforme sous jacent la partie périnéale de la vulve avec le vestibule ouvert, par écartement des petites lèvres. Je ne saurais trancher entre les deux lectures ; chacune pourra trouver des partisans.

Aucun autre tracé n'est visible sur cette face ornée, comme seins, ombilic ou membres supérieurs. La régularité de la section de la partie supérieure milite en faveur de l'absence initiale de tête. L'irrégularité de l'extrémité inférieure peut faire envisager que les cuisses ou les membres inférieurs ont pu être représentés à l'origine, mais deux arguments plaident contre cette hypothèse : l'espace inter-crural, marqué par le petit sillon fusiforme signalé (s'il n'est pas vulvaire), est très bref mais complet et n'a pas été amputé par une fracture éventuelle, et la crête corticale inférieure présente le même aménagement par sciage que la crête supérieure (fig. 6).

Les faces latérales ont été aplanies dans leur partie tronculaire, alors qu'elles gardent un relief arrondi dans la partie pelvi-crurale et elles portent sur les $2 / 3$ supérieurs un sillon longitudinal rectiligne étroit et peu profond, séparant la face ornée (recto) de l'autre face qui ne l'est pas (verso). C'est sur la face latérale droite qu'ont été tracés les trois arcs de cercle signalés, au dessus d'un trait gravé ténu, peu profondément incisé, intéressant uniquement le tronc, d'aspect similaire et se terminant au dessus de la hanche (fig. 7). On ne saurait y reconnaître une figure précise, mais la présence à l'extrémité du $3 e$ arc, contigu à la hanche droite, d'un tracé trifide, pourrait faire évoquer une nageoire caudale et une image pisciforme très schématique, et donner un sens particulier à la frise. Les traits légèrement gravés sur la hanche droite peuvent difficilement être interprétés (poisson ?) et il n'est pas sûr qu'ils soient anthropiques. Je suis tenté de rapprocher de ce segment de corticale de bois de renne le petit bâton perforé trouvé dans la couche sus-jacente, orné d'une vulve sur une face et qui offre trois images pisciformes sur les faces latérales et postérieure. Le poisson ne pouvait être ignoré de ces Magdaléniens de bord de Vézère, et il est effectivement représenté dans nombre de sites, le plus fameux étant l'abri gravettien du Poisson ! La face latérale gauche offre un sillon longitudinal plus marqué qu'à droite (fig. 8) et, sur ce tracé, se greffent d'autres traits, courts et obliques, de sens obscur ; dans le prolongement inférieur, mais avec un tracé oblique vers l'avant, se trouve le profond sillon du contour crural externe gauche mentionné plus haut (qui fait défaut à droite du corps féminin).

La discrétion de l'ornementation de ce segment cortical de bois de renne contraste avec ce qu'offrent de nombreux objets en os, ivoire et bois de renne découverts dans le site et publiés tant par Lartet et Christy que par Capitan et Peyrony : manifestement, ici, c'est la représentation du corps humain féminin le sujet principal destiné à retenir l'attention, et non le décor. Une autre particularité de cette figure féminine est son absence de symétrie, manifestée sur plusieurs points. Le premier est un décor différent sur chaque face latérale, une frise d'arcs à droite, un tracé linéaire « empenné » à gauche. Et puis, il y a la morphologie du massif pelvi-crural, avec la présence d'un détourage externe de la racine crurale gauche, la moindre ampleur de la hanche homolatérale, la situation légèrement plus basse du rebord iliaque et la plus grande longueur de la portion de cuisse figurée du même côté. Cela n'est pas sans évoquer une posture hanchée du corps, avec appui à droite et légère flexion vers l'avant et en dedans du membre inférieur gauche, une attitude que j'ai reconnue chez d'autres figures féminines, surtout gravettiennes (le «Torse » et le "Manche de poignard » de Brassempouy, la statuette de Péchialet, le "Losange » de Grimaldi), mais une seule figure magdalénienne (femme $\mathrm{n}^{\circ} 4$ de Rochereil), et qui participe au réalisme kinésique que j’ai défini (Duhard 1993).

Cet objet a-t-il été utilisé comme «poignard " ou " ciseau "? Rien n'est moins sûr : il est de petites dimensions, parait fragile et n'a pas de tranchant proximal ni distal. Rigaud (1984) emploie le terme de baguette en traitant de l'utilisation du ciseau dans le débitage du bois de renne. Ce terme de baguette me convient bien, correspondant d'ailleurs à la définition de Passemard (1916) : un « objet en matière osseuse à une face plane, qui comprend nombre de formes différentes ». Breuil et Robert (1951) ont décrit, sans donner de définition, des baguettes demi-rondes de la grotte de la Vache, qui offrent une face convexe et une face plane, comme l'objet présenté. II me semble donc que cette pièce de La Madeleine est une baguette semi-ronde, mais pour ne pas heurter les paléoostéologistes de la commission sur l'industrie osseuse (Camps-Fabrer 1988), je la baptiserai « pièce sur segment cortical de bois de renne, allongée et à section semironde ". Madame Feruglio conteste cette dénomination, ce que sa compétence autorise et mon ignorance excuse.

L'aspect lisse de sa surface corticale pourrait résulter, éventuellement, d'un usage de lissoir, servant à lustrer les cuirs, mais il y aurait, dans ce cas, un polissage plus marqué des surfaces en relief, ce qui n'est pas le cas. II ne me semble pas avoir été destiné à un rôle utilitaire, comme le galet pyramidal gravé de deux humains de même origine et portant des traces de compression, mais à un autre rôle, 
qui reste à imaginer : jouet ? (comme le « berceau » de Brassempouy, Delporte 1993, ou d'autres statuettes schématiques), fétiche ? amulette ou talisman ? Une seule certitude, il n'a pas été porté, étant dépourvu de perforation ou de traces de suspension et il ne semble pas avoir été utilisé comme outil.

\section{Conclusion}

Cette image de femme sculptée complète heureusement la petite série de figurations humaines de l'abri de La Madeleine, essentiellement présentes dans la Magdalénienne IV, et illustre cette évolution d'un style descriptif vers un style elliptique au long du Paléolithique, que j'ai soulignée à propos des figures féminines françaises (Duhard 1993). Elle trouve tout à fait sa place à côté des figures féminines schématiques de la même période, comme en offrent la Roche à Lalinde, la Gare de Couze, etc. Elle n'est pas sans évoquer une autre figure féminine magdalénienne, provenant de l'abri des Harpons à Lespugue (Haute-Garonne), méconnue par R. de SaintPérier, comme par G. Mortillet, et décrite par M. Allard (1988) : un corps féminin svelte, de face, est gravé en léger relief sur une baguette arrondie en bois de renne ${ }^{11}$; aussi sobrement tracée que cette femme de la Madeleine, elle est également sans tête ni membres supérieurs ou jambes, mais elle est porteuse de seins ptosés. Dans ma publication, j'avais suivi l'orientation donnée par M. Allard, mais il me semblerait plus judicieux de lire la silhouette féminine dans l'autre sens, celui adopté ici (fig. 9), où se voient deux seins d'inégale hauteur, un pelvis avec le « $V$ » pubo-géni- tal et le contour externe du membre inférieur gauche en adduction ; dans cette seconde lecture, le corps féminin représenté est également en posture hanchée.

Cette femme de La Madeleine offre également une ressemblance, à mes yeux, avec une statuette plus connue, provenant d'un site contemporain et voisin, situé lui aussi en bord de Vézère et en amont, la "Vénus impudique » de Laugerie-Basse, trouvée par P. de Vibraye en $1864^{12}$. Ce sujet d'aspect également svelte présente la même morphologie du tronc et du pelvis, et un triangle pubo-génital aussi développé en hauteur (fig. 10). Avec le retentissement qu'eût cette découverte, il est un peu surprenant que cette "lame de poignard" de La Madeleine n'ait pas été identifiée comme elle devait l'être, alors même que Louis Capitan était médecin et anthropologue ! Mais il est vrai que nul n'est parfait, même un médecin. Si j'avais à décrire le sujet ayant servi de modèle à cette figure féminine, je dirais qu'il s'agit d'une femme dépourvue d'adiposité, probablement n'ayant pas eu d'enfant, en bref une jeune Magdalénienne nulligeste et svelte, qui dut avoir plus tard une longue descendance.

Je me garderai bien de proposer une motivation à cette œuvre figurative féminine de La Madeleine mais ne peut m'empêcher de constater la présence dans ce site de représentations sexuées (femmes et hommes) et sexuelles (vulves et phallus), ce qui relève sinon d'une préoccupation reproductive, du moins d'un intérêt pour l'appareil génital, qui semble une constante chez l'humain, quelle que soit l'époque.

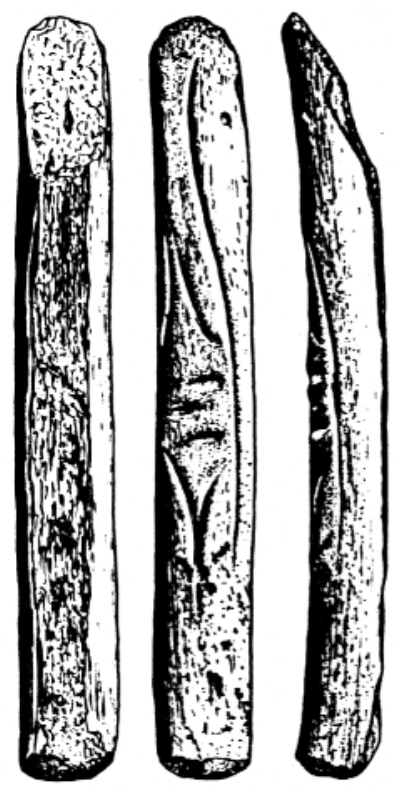

Figure 9 - La figure féminine de Lespugue (Allard 1988), $110 \mathrm{~mm}$ de long.

Figure 9 - Lespugue female figure (Allard 1988), $110 \mathrm{~mm}$ lenght.
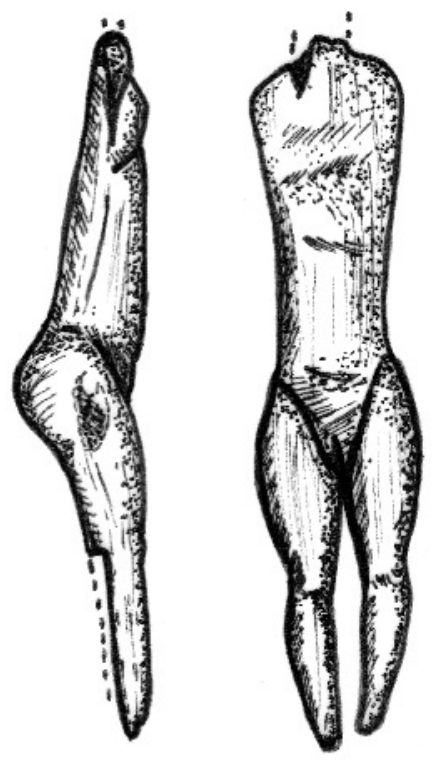

Figure 10 - La « Vénus impudique » de Laugerie-Basse (Duhard 1993), $77 \mathrm{~mm}$ de hauteur.

Figure 10 - "Venus impudique ", LaugerieBasse (Duhard 1993), $77 \mathrm{~mm}$ high.

(11) Longue de $110 \mathrm{mmn}$ la pièce est visible au musée municipal de Saint-Gaudens.

(12) Statuette en ivoire de $77 \mathrm{~cm}$ de haut, conservée au laboratoire de préhistoire du Musée de l'Homme (Réf. LPLB 381891372 ). 


\section{Remerciements}

J'exprime mes vifs remerciements à Madame Valérie Feruglio pour sa relecture, ses conseils et ses corrections, et à Madame Valérie Salcedo pour sa traduction en anglais.

\section{Références bibliographiques}

ALLARD M. 1988 - Une nouvelle représentation féminine magdalénienne à Lespugue (Haute-Garonne). Bulletin de la société préhistorique française, T. 85, 9, 83-85.

BOUVIER J.M. 1990 - Bases objectives de la chronologie de l'art mobilier paléolithique en Gironde, Périgord et Charente. In l'art des objets au Paléolithique, T 1, l'Art mobilier et son contexte, p. 65-75, Ministère de la Culture.

BREUIL H., ROBERT R. 1951 - Les baguettes demirondes de la grotte de la Vache (Ariège). Bulletin de la société préhistorique française, T. 48, n 9-10, p. 453-457.

CAMPS-FABRER H. (dir) 1988 - Fiches typologiques de l'industrie osseuse préhistorique : Biseaux et tranchants. Commission de nomenclature sur l'industrie de l'os préhistorique. Union internationale des sciences préhistoriques et protohistoriques. Treignes, CEDARC, 128 p., ill.
CAPITAN L., PEYRONY D. 1928 - La Madeleine. Son gisement, ses industries, ses oeuvres d'art. Paris : Librairie E. Noury, Publications de I'I.I.A., n 2, 70 fig., 19 pl.

DELPORTE H. 1993 - L'image de la femme dans l'art préhistorique. Paris : Picard, 1 vol. (2e édition).

DUHARD J.-P. 1993 - Réalisme de l'image féminine paléolithique. Paris : CNRS, Cahiers du quaternaire $n^{\circ} 19$. 1 vol. III. (publication de la thèse de 1989).

LARTET E., CHRISTY H. 1865-1875 - Reliquiae aquitanicae ; being contributions to the Archaeology and the Palaeontology of Perigord and the adjoining provinces of southern France. London : Thomas Ruppert Jones \& c., 302 \& 204 p., 87 plates, 8 maps, 182 woodcuts (consulté sur le site

http://www.archive.org/stream/reliquiaeaquitan00lartuoft\# page/n37/mode/2up).

PASSEMARD E. 1916 - Sur les baguettes demi-rondes. Bulletin de la société préhistorique française, T. $13, \mathrm{n}^{\circ} 6$, p. 201-307.

RIGAUD A. 1984 - Utilisation du ciseau dans le débitage du bois de renne à la Garenne-Saint-Marcel (Indre). Gallia préhistoire, T. 27, fascicule 2, p. 245-253. 
